\title{
On the fractal structure of galaxy distribution and its implications for cosmology
}

\author{
Yurij V. Baryshev \\ Astronomical Institute of the Saint-Petersburg University, 198904, St.-Petersburg, Russia \\ Francesco Sylos Labini ${ }^{1}$, Marco Montuori, Luciano Pietronero \\ Dipartimento di Fisica, Università di Roma "La Sapienza" P.le A. Moro 2, I-00185 Roma, Italy. \\ INFM, Sezione di Roma 1

\section{Pekka Teerikorpi} \\ Tuorla Observatory, University of Turku, FIN-21500 Piikkiö, Finland
}

\begin{abstract}
Two fundamental empirical laws have been established in the analysis of galaxy space distribution. First, recent analyses have revealed that the three dimensional distribution of galaxies and clusters is characterized by large scale structures and huge voids: such a distribution shows fractal correlations up to the limits of the available samples. This has confirmed the earlier de Vaucouleurs power-law density - distance relation, now corresponding to a fractal structure with dimension $D \approx 2$, at least, in the range of scales $\sim 1 \div 200 \mathrm{Mpc}\left(H_{0}=55 \mathrm{~km} / \mathrm{sec} / \mathrm{Mpc}\right)$. An eventual cut-off towards homogenization has not been yet identified. Second, since Hubble's discovery, the linear redshift-distance law has been well established within $200 M p c$ and also much deeper. The co-existence of these laws within the same scales is a challenge for the standard cosmology where the linear Hubble law is a strict consequence of homogeneity of the expanding universe. This puzzle is now sufficiently strong to raise doubts for the standard cosmology.
\end{abstract}

${ }^{1}$ Dépt. de Physique Théorique, Université de Genève, 24, Quai E. Ansermet, CH-1211 Genève, Switzerland 


\section{INTRODUCTION}

The basic assumption of the standard Big Bang cosmology is the Einstein's Cosmological Principle which, in fact, is the hypothesis that the universe is spatially homogeneous and isotropic on large scales (see e.g. Weinberg, 1972; Peebles, 1993). Homogeneity of matter distribution plays the central role in the expanding universe model, because it implies that the recession velocity is proportional to distance. This means that linear velocity-distance law $v_{\exp }=H l$, identified by the observed Hubble law, is valid at scales where matter distribution can be considered on average uniform.

For a long time, astronomers used 2-dimensional photographic plates of the sky as the basic means for the galaxy structures studies without no direct observations of the 3-dimensional large-scale matter distribution. Then the linear Hubble law inferred from redshift measurements at various distances, was interpreted as indirect evidence for average homogeneity at the same scales (see Peebles, 1993).

Recently, several 3-dimensional maps of galaxy distribution have become available, based on massive redshift measurements. Surveys such as CfA, SSRS, Perseus-Pisces, IRAS, LEDA, APM-Stromlo, Las Campanas, and ESP for galaxies, and Abell and ACO for galaxy clusters have detected remarkable structures such as filaments, sheets and voids. The galaxy maps now probe scales up to $200 M p c$ and they show that the large-scale structures are common features of the local universe. Fig.1 illustrates this change from flat sky vault to the deep 3-dimensional space.

Pietronero and collaborators (see the review by Sylos Labini et al. ,1998 for a comprehensive discussion of the subject) by using the methods of modern statistical physics, have shown that, in the above mentioned surveys, galaxy distribution exhibits fractal behavior with dimension $D \approx 2$ at least up to $200 M p c$. This

implies that, in the context of the standard model, one would expect strong deflections from the linearity of the redshift-distance relation in the range of scale where the inhomogeneities are present. However, observations show an almost strictly linear Hubble law at the same distance scales where fractal distribution has been established. In this article we explore this unexpected inconsistency of the standard model.

\section{DE VAUCOULEURS LAW AND UNIVERSAL FRACTAL STRUCTURE}

In the history of astronomy, parallel to the idea of homogeneity which was adopted by Newton, the alternative view of a hierarchic structure of the Universe has existed since it was created by Swedenborg, 
Kant and Lambert in the 18th century. They imagined the majestic picture of the hierarchy of stars forming clusters forming galaxies which in turn are clustered into yet larger systems and so on, either indefinitely (Swedenborg, Kant) or stopping at some level (Lambert). This idea was revived by Fournier d'Albe and Charlier who discussed a hierarchy where the mass within radius $r$ varies as $M \sim r$ (for historical reviews see Mandelbrot, 1977 and Baryshev \& Teerikorpi, 1998a).

De Vaucouleurs (1970) studied the possibility, firstly mentioned by Carpenter (1938) and Kiang (1967), that there is a universal density-radius power law as a basic factor in cosmology, reflecting a hierarchic distribution. He suggested the law:

$$
\rho(r)=\rho_{l}\left(\frac{r}{r_{l}}\right)^{-\gamma}
$$

where $\rho(r)$ is the mass density in the sphere of radius $r, \rho_{l}$ and $r_{l}$ are the density and radius at the lower cutoff of the hierarchy, and $\gamma$ is nowadays called the fractal co-dimension or correlation exponent. This is related to fractal dimension $D$ as:

$$
\gamma=3-D
$$

The concept of fractal was introduced by Mandelbrot (1977). The mathematical framework of fractal geometry allows one to study in a quantitative way the properties of irregular systems. Fractal structures are characterized by clusters and voids at all scales, and they are intrinsically non-analytical: their scaling behavior is described by power law functions. The density-radius relation (Eq.1) is an example of such a behavior.

It is a remarkable property of fractal structure that the power law scaling for the density (Eq.1) holds for any structure point. This means that the average density decreases not only away from us, but from any other galaxy. This ensures the statistical equivalence of all the observers in the distribution, which is required by any reasonable cosmological principle (see below).

In a fractal structure the conditional (average) density from an occupied point behaves as (Pietronero 1987)

$$
\Gamma(r)=S(r)^{-1} \frac{d N(r)}{d r}=\frac{D}{4 \pi} B r^{-(3-D)}
$$

where $S(r)$ is the area of a spherical shell of radius $r$, thickness $d r$ and $d N(r) / d r$ is the number of points in the shell and $B$ is constant related to the lower cut-off (see Eq.11). 
If the distribution is fractal up to a certain distance $\lambda_{0}$, and then it becomes homogeneous, we have that:

$$
\begin{gathered}
\Gamma(r)=\frac{B D}{4 \pi} r^{D-3} \quad r<\lambda_{0} \\
\Gamma(r)=\frac{B D}{4 \pi} \lambda_{0}^{D-3}=\text { const. } r \geq \lambda_{0}
\end{gathered}
$$

From Eq.11 it results that the conditional density is the appropriate statistical tool able to study scale invariant systems as well as homogenous ones. On the other hand, the standard correlations analysis is strongly based on the assumption that the system has reached homogeneity in the sample analyzed. In fact, the standard approach (Peebles, 1990; Davis \& Peebles, 1983) identifies a length-scale, called the "correlation length" $r_{0}$, and defined by the relation:

$$
\xi\left(r_{0}\right)=1
$$

where

$$
\xi(r)=\frac{<n\left(\overrightarrow{r_{*}}\right) n\left(\overrightarrow{r_{*}}+\vec{r}\right)>}{<n>^{2}}-1
$$

is the (standard) two point correlation function. (In Eq.6 $n(\vec{r})$ is the local number density). The basic point that Pietronero and collaborators (Pietronero, 1987; Coleman \& Pietronero, 1992; Sylos Labini, et al. 1998) stressed, is that the mean density, $\langle n\rangle$, used in the normalization of $\xi(r)$ (Eq.6), is not a well defined quantity in the case of self-similar distribution and it is a direct function of the sample size. Hence only in the case that the homogeneity has been reached well within the sample limits, the $\xi(r)$-analysis is meaningful, otherwise the a priori assumption of homogeneity is incorrect and the characteristic lengths, like $r_{0}$, became spurious.

For example the expression of the $\xi(r)$ in the case of fractal distributions is

$$
\xi(r)=\left(\frac{3-\gamma}{3}\right)\left(\frac{r}{R_{s}}\right)^{-\gamma}-1
$$

where $R_{s}$ is the depth of the spherical volume where average density is computed. From Eq.77 follows that:

i.) the so-called correlation length $r_{0}$ (defined as $\xi\left(r_{0}\right)=1$ ) is a linear function of the sample size $R_{s}$ :

$$
r_{0}=\left(\frac{3-\gamma}{6}\right)^{\frac{1}{\gamma}} R_{s}
$$


and hence it is a spurious quantity without physical meaning: it is simply related to the sample finite size.

ii.) $\xi(r)$ is power law only for:

$$
\left(\frac{3-\gamma}{3}\right)\left(\frac{r}{R_{s}}\right)^{-\gamma} \gg 1
$$

hence for $r \ll r_{0}$ : for larger distances there is a clear deviation from the power law behavior due to the definition of $\xi(r)$. This deviation, however, is just due to the size of the observational sample and does not correspond to any real change of the correlation properties. It is clear that if one estimates the exponent of $\xi(r)$ at distances $r \lesssim r_{0}$, one systematically obtains a higher value of the correlation exponent due to the break of $\xi(r)$ in the log-log plot (see for more details Sylos Labini et al. , 1998).

The analysis performed by $\xi(r)$ is therefore mathematically inconsistent, if a clear cut-off towards homogeneity has not been reached, because it gives an information that is not related to the real physical features of the distribution in the sample, but to the size of the sample itself.

The new approach proposed by Pietronero and collaborators has been applied to the above mentioned redshift surveys, with the result that fractal structures extend to scales larger than $\sim 200 M p c$. In particular, the correlation function has been found to have a power law behavior up to distance $\sim 300 M p c$, establishing that Eq.11 holds with $\gamma=1 \pm 0.2$, or in terms of fractal dimension $D=2 \pm 0.2$. The eventual cut-off towards homogeneity (the length scale $\lambda_{0}$ in Eq.⿴囗十) has not been yet identified. The long-range correlations detected are limited only by the size of the available catalogs. The results from the $\Gamma(r)$ function analysis in the presently available redshift surveys are given in Tab.] (from Pietronero et al. , 1997).

Usually analysis of the space distribution is based on distances estimated from redshifts which always include a non-distance part due to peculiar velocities of galaxies. Recently, Teerikorpi et al. (1998) have derived the radial galaxy spatial distribution around our Galaxy, utilizing over 5000 Tully-Fisher photometric distances from the KLUN program (KLUN = Kinematics of the Local Universe). First results give evidence for a decrease in the average density consistent with the fractal dimension $D \approx 2.2$ in the distance range $20 \div 200 M p c$. This may be regarded as a new, independent confirmation of the results of Sylos Labini et al. (1998). 


\section{HUBBLE LAW}

In his classic paper, Hubble (1929) found a roughly linear relation between the spectral line displacement $z=\left(\lambda_{o b s}-\lambda_{e m}\right) / \lambda_{e m}$ of the line emitted by a far away galaxy $\lambda_{e m}$, and its distance $r$. The empirical Hubble Law may be written as

$$
c z=H_{0} r
$$

where $c$ is the velocity of light and $H_{0}$ is the Hubble constant. As an observationally established relation, the Hubble law does not refer to any interpretation of redshift, though Hubble had in mind especially the de Sitter effect (Smith, 1979). This effect in the static space, as well as the space expansion and Doppler mechanisms for redshift, yields at first order to Eq.10. If redshift is interpreted as a motion effect, then

$$
V \approx H_{0} r
$$

where $V$ is either space expansion velocity $v_{\text {exp }}$ or ordinary velocity of a body moving in the Euclidean space. Usually this velocity-distance relation is called the Hubble Law, but it is more correct to regard it as the redshift-distance relation of Eq.10 (Harrison, 1993). This is based on the primarily measured quantities (redshift and distance), while velocity is inferred from redshift in the frame of some cosmological model.

Since its discovery, the validity of the Hubble law has been confirmed in an ever increasing distance interval where local and more remote distance indicators may be tied together. Recently, several new distances have been measured to local galaxies using observations of Cepheid variable stars, thanks to the Hubble Space Telescope programmes (Freedman, 1996; Tammann, et al. 1996). Along with previous Earth-based Cepheid distances, methods like Supernovae Ia and Tully-Fisher have been better calibrated than before and confirm the linearity with good accuracy up to $z \approx 0.1$. Brightest cluster galaxies trace the Hubble law even deeper, up to $z \approx 1$, and radio galaxies have provided such evidence at still larger redshifts (Sandage, 1995).

It is well known that there are small deviations $\delta V$ from the Hubble velocity $V_{H}$, connected with local mass concentrations such as the Virgo Cluster, and, possibly the Great Attractor. However, these perturbations are still only of the order $\delta V / V_{H} \sim 0.1$, while in the general field the Hubble law has been suggested to be quite smooth, with $\delta V$ around $50 \mathrm{~km} / \mathrm{s}$ (Sandage, 1995; Karachentsev \& Makarov, 1996). 
The observed Hubble law is derived for our Galaxy as the center, and it does not necessarily imply that it is observed similarly from other galaxies. However, if our Galaxy is not in a privileged position in the Universe, then one must conclude that the Hubble law is universal. The small scatter even at the small distances of a few Mpc also seems to be a general feature: the structure and kinematics of our Local Group is typical of several other small groups (Karachentsev, 1996; Governato et al. , 1997).

There have been proponents of global non-linearity of the Hubble law, which is expected in some non-standard cosmological models (e.g. Segal, 1976). However, it has been convincingly shown that such deviations from linearity are easily produced by a statistical distortion effect known as the Malmquist bias in magnitude limited samples. When one by-passes this problem using suitable methods, the linearity is recovered (Teerikorpi, 1997). The second main advancement is concerned with measurement of the value of the Hubble constant $H_{0}$. Since Hubble's $559 \mathrm{~km} / \mathrm{s} / \mathrm{Mpc}, H_{0}$ has decreased and according to the most recent studies (Tammann et al. , 1996; Theureau et al. , 1997), seems to have stabilized around $55 \mathrm{~km} / \mathrm{s} / M p c$. In this article, we shall use this value for distance estimates from redshift.

Without actual knowledge of the matter distribution, the linearity and small scatter of the observed Hubble law for field galaxies would make one easily guess that the galaxies are uniformly distributed: as it was asserted above, this is the basis for the linear Hubble law in the standard cosmology. In fact, it has been a common supposition that when the Hubble law was found in the nearby space, one finally had entered a cosmologically representative region of the Universe. At the same time, it has been clear that at small distances where Hubble found his relation, the galaxy distribution is quite inhomogeneous. Though, it has been believed that beyond some, not too large distance, the distribution should become uniform.

\section{THE HUBBLE - DE VAUCOULEURS DIAGRAM}

As we have already mentioned, studies of the 3-dimensional galaxy universe have shown that de Vaucouleurs' prescient view on the matter distribution is valid at least in the range of scales $\sim 1 \div 200 M p c$. The Hubble and de Vaucouleurs laws describe very different aspects of the Universe, but both have in common universality and observer independence. This makes them fundamental cosmological laws and it is important to investigate the consequences of their coexistence at similar length-scales. In Fig.2 we display these laws together. 
A representative Hubble law has been taken from Fig.4 of Teerikorpi (1997), based on Cepheid distances to local galaxies, Tully-Fisher distances from the KLUN programme, and Supernovae Ia distances. The behavior of the conditional density (De Vaucouleurs law) presented in Fig.2 2 was taken from Sylos Labini et al. (1998).

The puzzling conclusion from Fig.2 is that the strictly linear redshift-distance relation is observed deep inside the fractal structure. This empirical fact presents a profound challenge to the standard model where the homogeneity is the basic explanation of the Hubble law, and "the connection between homogeneity and Hubble's law was the first success of the expanding world model" (Peebles et al. , 1991). This also reminds us the natural reaction of several authors: "In fact, we would not expect any neat relation of proportionality between velocity and distance [for such close galaxies]" (Weinberg, 1977).

However, contrary to the expectations, modern data show a good linear Hubble law even for nearby galaxies. How unexpected this actually is, can be expressed quantitatively for the standard model and is discussed below.

\section{IMPLICATIONS FOR FRIEDMANN MODELS}

\subsection{Homogeneity and isotropy as the basis for Hubble law}

According to the standard Big Bang model the universe obeys to the Einstein's Cosmological Principle: it is homogeneous, isotropic and expanding (Weinberg, 1972; Peebles, 1988; 1993). Homogeneity of matter distribution is the central hypothesis of the standard cosmology because it allows one to introduce the space of uniform curvature in the form of the Robertson-Walker line element

$$
d s^{2}=c^{2} d t^{2}-R(t)^{2}\left[d y^{2}+f(y)^{2}\left(d \Theta^{2}+\sin ^{2} \Theta d \Phi^{2}\right)\right]
$$

Here $y, \Theta, \Phi$ are comoving space coordinates, $t$ is comoving cosmic time, $f(y)=\sin (y), y, \sinh (y)$ for curvature-constant values $k=1,0,-1$ respectively, $R(t)$ is the scale factor, and $c$ is the velocity of light. This line element leads immediately to a linear relation between velocity and proper distance (Robertson, 1955). Indeed, consider a comoving body at a fixed coordinate distance from a comoving observer. At cosmic $t$, let $l=R(t) y$ be the proper distance from the observer. The expansion velocity $v_{\text {exp }}=d l / d t$, defined as the rate of change of the proper distance $l$, is 


$$
v_{\text {exp }}=H l=c \cdot \frac{l}{l_{H}}
$$

where $H=\dot{R} / R$ is the Hubble constant and $l_{H}=c / H$ is the Hubble distance. In this way, the linear velocity-distance relation of Eq.13 is an exact formula for all Friedmann models and a rigorous consequence of spatial homogeneity. In particular, for $l>l_{H}$, the expansion velocity $v_{\text {exp }}>c$. Such an apparent violation of special relativity is consistent with general relativity (Harrison, 1993).

In the expanding space the wavelength of an emitted photon is progressively stretched, so that the observed redshift $z$ is given by Lemaitre's redshift law

$$
z=\frac{R\left(t_{o b s}\right)}{R\left(t_{\text {em }}\right)}-1
$$

which is a consequence of the radial null-geodesic of the line element Eq.12. For $z \ll 1$ Eq.14 yields $z \approx d R / R \approx H_{0} d t \approx l / l_{H}$, and from Eq.[13 one gets the approximate velocity-redshift relation that is valid for small redshifts

$$
v_{e x p} \approx c z
$$

The relations between $v_{\text {exp }}, l$, and $z$ have been recently discussed in an especially clear manner by Harrison (1993) who emphasized that the expansion velocity-redshift relation differs from the relativistic Doppler effect. So, the space expansion redshift mechanism in the standard model is quite distinct from the usual Doppler mechanism. We stress this points, because in the literature these two redshift mechanisms are often confused.

In the context of the standard cosmology, it has been natural to interpret the Hubble Law as a reflection of Eq.11 and Eq.15 and to regard the coefficient of proportionality $H_{0}$ in Eq.10 as the present value of the theoretical Hubble constant $H$ from Eq.13.

The behavior of the scale factor $R(t)$ is governed by the Einstein equations which describe how the geometry of space changes under the influence of a mass distribution. In the case of homogeneity and zero-pressure, they lead to the Friedmann Equation:

$$
\frac{d^{2} R}{d t^{2}}=-\frac{4 \pi}{3} G R \rho
$$

This equation gives two basic parameters of the Friedmann models: the Hubble parameter $H(t)=$ $[d R(t) / d t][1 / R(t)]$, the value of which at the present time $t_{0}$ is equal to the Hubble constant $H_{0}$, and the density parameter $\Omega=\rho / \rho_{\text {crit }}$ where the critical density is defined as $\rho_{\text {crit }}=3 H^{2} / 8 \pi G$. 
Note that the Friedmann Equation coincides exactly with the equation for the Newtonian force acting on the unit mass on the surface of a spherical ball with uniform density $\rho(t)$ and radius $R(t)$. Hence, there is a tight analogy between the behavior of the scale factor in Friedmann models and the expanding radius of the finite Newtonian ball. This analogy was first noticed by Milne (1934) and later discussed by Layzer (1954) and McCrea (1955).

\subsection{Gravitational growth of density fluctuations}

In order to give a rough estimation of the gravitational growth of density fluctuations and accompanying deflection from the linear velocity-distance relation, one can utilize the linear perturbation (LP) approximation (see Peebles, 1980; 1993).

We consider now the case of an expanding universe, where an average density is well defined and has a constant value $\rho_{0}$. In such a case, by neglecting relativistic effects and the terms depending on pressure, according to the linear approximation, there is a velocity deflection $\delta V$ from the unperturbed Hubble flow $V_{H}=H_{o} r$ in the scale where the density perturbation is $\delta \rho$. In the case of zero cosmological constant and spherical mass distribution, this deflection has grown during the Hubble time to the present value which is (Eq.20.55 from Peebles, 1993):

$$
\frac{\delta V}{V_{H}}=\frac{1}{3} \Omega_{0}{ }^{0.6} \frac{\delta \rho}{\rho_{0}}
$$

where $\Omega_{0}=\rho_{0} / \rho_{\text {crit }}$ is the density parameter of the Friedmann model. This approximation holds in the limit $\delta \rho / \rho_{0} \ll 1$.

Let us consider a two-component model for the density distribution in a Friedmann universe. First, there is the component which exhibits fractal behavior up to a maximum scale, and which we call $\lambda_{0}$. At larger scales this component is homogeneous with an average density $\rho_{\text {lum }}$. The second component is dark matter, homogenous at all scales, with density $\rho_{\text {dark }}$. For such a model there is a definite constant density at all scales larger than $\lambda_{0}$. This density $\rho_{0}$ is the sum of $\rho_{\text {lum }}$ and $\rho_{\text {dark }}$. This means that the behavior of this model at scales larger than $\lambda_{0}$ is identical to that of the Friedmann model for which

$$
\Omega_{0}=\Omega_{\text {dark }}+\Omega_{\text {lum }}
$$


At such large scales the Hubble law is unperturbed. The density distribution of luminous matter for scales $r<\lambda_{0}$, can be written as

$$
\rho_{l u m}(r)=\rho_{\text {lum }}\left(\frac{\lambda_{0}}{r}\right)^{\gamma}
$$

where $\gamma=3-D$ as usual. For the scales $r \geq \lambda_{0}$ we have that

$$
\rho_{\text {lum }}(r)=\rho_{\text {lum }}=\rho_{\text {lum }}\left(\lambda_{0}\right)
$$

The density contrast can be written as

$$
\frac{\delta \rho}{\rho_{0}}=\frac{\rho_{\text {lum }}(r)+\rho_{\text {dark }}-\rho_{0}}{\rho_{0}}
$$

In terms of the Friedmann density parameters as defined above, this becomes, at scales smaller than $\lambda_{0}$ :

$$
\frac{\delta \rho}{\rho_{0}}=\frac{\Omega_{\text {lum }}}{\Omega_{0}}\left(\left(\frac{r}{\lambda_{0}}\right)^{-\gamma}-1\right) .
$$

At scales larger than $\lambda_{0}$ the density contrast clearly vanishes. A useful distance scale can be defined by the relation

$$
\frac{\delta \rho\left(\lambda_{0.5}\right)}{\rho_{0}}=0.5
$$

At scale larger than $\lambda_{0.5}$ we have that $\delta \rho / \rho_{0} \ll 1$ and Eq.17 holds. From Eq.23 we easily obtain

$$
\lambda_{0.5}=\lambda_{0}\left(1+\frac{1}{2} \frac{\Omega_{0}}{\Omega_{\text {lum }}}\right)^{-\frac{1}{\gamma}} \text {. }
$$

By using the linear approximation (Eq.17), we may obtain a rough estimation of the expected deflection from the Hubble law in the two component model, for the scale in which the density contrast is less than 1. Although the linear approximation is valid only for $\delta \rho / \rho_{0} \lesssim 1$, the obtained results give a first quantitative indication of the effects of self-similar fluctuations. Moreover the assumption of spherical mass distribution is a rough one, and it holds only for average quantities. In the case of real fractals deviation from spherical symmetry can play an important role, at least at small scale, (Sylos Labini et al. , 1998c). Under these approximations, the radial velocity measured by an average observer at scale $r<\lambda_{0}$ is (from Eq. [17)

$$
V_{\text {obs }}=V_{H}\left(1-\left(\frac{1}{3}\right) \Omega_{0}{ }^{-0.4} \Omega_{\text {lum }}\left(\left(\frac{r}{\lambda_{0}}\right)^{-\gamma}-1\right)\right)
$$


Actually, this is the prediction averaged over many observers in different fractal structure points (galaxies). For any particular observer, there will be a deflection from this average law.

We take the maximum scale $\lambda_{0}$ of fractality and the fractal dimension $D$ from the observed cosmological de Vaucouleurs Law and calculate the expected deflections from the Hubble law in our two-components Friedmann model.

In Fig. 3 we show three theoretical predictions for the velocity deflection in the case where the observed fractal structure contains all the matter, i.e. when $\Omega_{l u m}=\Omega_{0}$. We have fixed $\lambda_{0}=200 M p c$ and fractal dimension $D=2$. In this case the linear approximation (from Eq.24) holds for $r \gtrsim 130 M p c$. At smaller scales we should consider non-linear effects which are not simple to be treated. However we should expect even stronger velocity perturbations, due to the highly inhomogeneous structures distribution. The predictions correspond to three values of the cosmological density parameter $\Omega_{0}=1,0.1,0.01$. From Fig.3 it follows that such Friedmann models, purely fractal within $200 M p c$, are excluded if $\Omega_{0} \gtrsim 0.01$. This confirms the previous suggestions that small $\Omega_{0}$ is needed for hierarchic models (Wertz, 1971; Haggerty \& Wertz, 1972, and Sandage, et al. 1972 - hereafter STH). Fang et al. (1991) used a similar two-components model as a possible explanation for claimed differences in the values of the Hubble constant as derived from near and distant galaxies. Because new data confirm the linearity of the Hubble law, implications of the two-component model are different.

For instance, there is a possibility to save the Friedmann universe with the critical density parameter $\Omega_{0}=1$. It was implied already by STH that dark matter, uniformly filling the whole universe and decreasing the relative density fluctuations, could reconcile the observed fractal structure with the linear Hubble law. However, they did not give a quantitative estimate of the amount of dark matter needed. With the new data on the Hubble and de Vaucouleurs laws, we can derive the lower limit for the amount of the needed uniform dark matter. In Eq.25 we fix $\Omega_{0}=1$ and let $\Omega_{\text {lum }}$ have different values. Fig.4 gives the developed version of the STH test, now showing that $\Omega_{\text {dark }}$ should be larger than 0.99 . If the actual maximum scale of fractality is larger than $200 M p c$ (with $D=2$ ), then the amount of luminous matter may be in conflict with the Big Bang nucleosynthesis prediction for baryonic matter. For example, if $\lambda_{0} \gtrsim 1000 M p c$ (as suggested by Sylos Labini et al. , 1998) then $\Omega_{\text {lum }}$ will be probably less than 0.001 .

It should be emphasized that this estimate of the amount of dark matter is independent on the physics of the early universe. It also does not depend on the determination of mass-to-luminosity ratio of galaxies. 


\section{DISCUSSION}

The surprising coexistence of the Hubble and de Vaucouleurs laws contains many still unexplored cosmological implications. Above we have discussed its importance from the viewpoint of the standard model where the Hubble law is more fundamental and requires homogeneity. In this case, there must be a homogeneously distributed dark matter, producing the linear Hubble law, and the observed fractal structure is confined to the sparse luminous matter having its properties (fractal dimension and maximum scale) due to some initial conditions.

It is also possible that the HdeV-diagram gives rise to a whole new direction in cosmological research, based on the more general Conditional Cosmological Principle (see below). In this case, the de Vaucouleurs law is as fundamental as the Hubble law and encompasses all matter. Then the Hubble law is not related to homogeneity, rather to isotropy. A new theoretical problem appears concerning the physical reason for the observationally revealed universal value of $D \approx 2$. We point out that the old question on the gravitational part of the cosmological redshift needs to be reanalyzed in the context of fractal structures.

Isotropic fractals allow the universe to be isotropic and inhomogeneous, without sacrificing the equivalence of all the observes. In this case it is possible to formulate a weaker version of the Cosmological Principle, called the Conditional Cosmological Principle (Mandelbrot, 1977; 1998; Coleman \& Pietronero, 1992). This implies an asimmetry between space points occupied by the structure and empty points. In

fact, a fractal structure is statistically the same from every point of the system. Such a property correspond to Local Isotropy rather than to homogeneity.

Could it be an accident that the observed fractal dimension for galaxy distribution is so close to 2 ? Perhaps there is some physical significance in the $D \approx 2$ fractal structure.

\subsection{Gravitational part of cosmological redshift}

A fully self-consistent dynamics of a fractal cosmology has not yet been developed. However, one can at least suggest that a Hubble law due to expansion is not expected, because of the global non-uniformity. Hence, if the linear Hubble law is, however, observed, its origin may be dominated by something other than expansion.

Gravitation is the only known mechanism, other than Doppler effect and space expansion, to produce 
the redshift of galaxy spectra. Bondi (1947) showed that the cosmological redshift may be divided in two parts, due respectively to relative motion and to gravitation effect. He emphasized that the spectral shift depends not only on the motion of source and observer, but also on the global matter distribution around the source, extending up to the observer. The gravitational part of the cosmological redshift was also considered by Zeldovich and Novikov (1984). They asserted that the gravitational shift in the homogeneous universe should increase as the square of distance, hence it does not obey the linear Hubble law. Furthermore, they concluded that it is a blueshift and not a redshift. This followed from their choice of a coordinate system, with the origin at the observer and the light sources rested on the surface of a sphere around the observer.

However, this choice is not unique. If one puts the origin on the source and the observer is on the surface of sphere surrounding the source, one similarly derives redshift. In favor of the last choice, one can present a causality argument: the emission of a photon by the source precedes its absorption by observer's light detector. After time $\tau$ from the emission of a photon, all observers at the surface of the sphere with radius $c \tau$, around the source, have a probability to detect the photon. Hence the shift will be redshift. The sign of the gravitational shift may be also extracted from the general Mattig's relation between proper distance and cosmological redshift, and it corresponds to redshift (Baryshev et al. , 1994).

It has been pointed out by Baryshev (1981) that inside a hierarchical structure with fractal dimension $D=2$, the gravitational part of the cosmological redshift is linearly proportional to distance, producing a linear $\left(z_{g r}-r\right)$ relation. Indeed, if $\rho(r) \sim 1 / r$, then the mass is spherical sample of radius $r$ scales as $M \sim r^{2}$, while the gravitational potential behaves as $\phi(r) \sim M / r \sim r$. So the gravitational redshift is $z_{g r} \sim \phi / c^{2} \sim r$. In this case the gravitational fractal contribution to the value of the Hubble constant is:

$$
H_{\text {grav }}=2 \pi G \rho_{l} r_{l} / c
$$

where $\rho_{l}$ and $r_{l}$ are the density and radius of the lower cut-off in the fractal structure (see Eq.11), $G$ is the gravitational constant, and $c$ is the velocity of light.

The gravitational part may be unobservable small or a significant fraction of the observed Hubble constant, depending on the product $\rho_{l} \cdot r_{l}$ which characterizes the fractal structure. As a convenient reference value, one can use $\rho_{l} r_{l}=1 / 2 \pi \mathrm{gcm}^{-2}$, in which case $H_{\text {grav }}=G / c=69 \mathrm{~km} / \mathrm{s} / \mathrm{Mpc}$. For an average galaxy with $\rho_{l}=5 \cdot 10^{-24} \mathrm{gcm}^{-3}$ and $r_{l}=10 \mathrm{kpc}$ this condition is fulfilled. 
It should be noted that the cosmological tests proposed for determining the nature of the observed redshift, such as independence from wavelength, Tolman surface brightness test, time delay, blackbody temperature (see Sandage, 1995), cannot distinguish between expansion and gravitational redshifts (Baryshev et al. , 1994). The astrophysical possibilities to detect this gravitational redshift will be discussed elsewhere by Baryshev \& Teerikorpi(1998b).

\subsection{Theoretical approaches to $D \approx 2$}

We briefly summarize various theoretical approaches which have considered the fractal properties of matter distribution.

An interesting analysis leading to $D \approx 2$ was made by Schulman and Seiden (1986). They proposed a model of galaxy formation. In their model the original process of galaxy formation occurs in analogy to the process of propagating star formation within our Galaxy. The birth of one galaxy stimulates the birth of nearby galaxies from the primeval cosmic gas. If this process occurs near its percolation threshold then a hierarchical structure is created. The critical fractal dimension for this process is quite close to 2 (about $1.95)$.

It is also interesting to note that within the so-called plasma cosmology originated by H. Alfven there is a possibility to understand the existence of hierarchic structures starting from certain general principles. Here the fractal dimension 2 appears as a consequence of electromagnetic processes in plasma (Lerner 1986).

Recently, in a paper by De Vega et al. (1996a) (see also De Vega et al. , 1996b; 1998) it was shown that gravitating mass points in statistical equilibrium are distributed with fractal dimension close to 2. Note that the energy density of the gravitational field (which is positive and localizable in the field theory - Baryshev 1996) will be homogeneous for fractal structures with $D=2$. This may be a way to understand the above result on the equilibrium state of self-gravitating point mass distribution having $D \approx 2$.

\section{CONCLUSIONS}

Investigation of the large scale distribution of galaxies in the universe is now in a new phase, which is characterized by new observational data and new methods of analysis. It has become an especially hot 
and debated topic in cosmology, because the revealed fractality contradicts Cosmological Principle in the sense of homogeneity. Above we have discussed the fractality and its implications for cosmology. Our main conclusions are:

- Observations show that there is a fractal distribution of galaxies, having fractal dimension $D \approx 2$ in the scale range from $1 M p c$ to, at least, $200 M p c$. While there is a general agreement on the small scale fractal properties of galaxy distribution, the actual value of $D$ and the eventual presence of an upper cut-off, are still matter of debate (see e.g. Pietronero et al. , 1997; Davis, 1997; Guzzo, 1997; Coles, 1998; Sylos Labini et al. , 1998a; 1998b).

- The traditional statistical analysis based on the assumption of homogeneity (i.e. $\xi(r)$ ), should be replaced by the more general methods of modern statistical physics. Such methods are able to characterize scale-invariant distributions as well as regular ones.

- An isotropic fractal distribution is fully compatible with the reasonable requirement of the equivalence of all the observers. Hence the Standard Cosmological Principle, which requires isotropy and homogeneity, may be replaced by the Conditional Cosmological Principle. In such a case the condition of local isotropy around any structure point, without the assumption of analyticity of matter distribution, does not imply the homogeneity of matter distribution.

- The paradox of linear Hubble law within the fractal de Vaucouleurs density-distance law is sharpened with the new data: strong deflections from the Hubble flow are expected in the framework of the standard Friedmann model.

- From a developed version of the old Sandage-Tammann-Hardy test we derive the minimum amount of the uniform dark matter, $\Omega_{\text {dark }}=0.99$, which is consistent with the presently known Hubble and de Vaucouleurs laws. This result is independent of the early universe physics. If the maximum scale of fractality is larger than $200 M p c$, this test may be regarded as crucial for the standard cosmology.

- It is noted that within fractal structure there is a net gravitational redshift which follows a linear redshift - distance law for fractal dimension 2. The significance of its contribution to the cosmological redshift depends on the amount of mass coupled to fractals and requires further study. 


\section{Acknowledgements}

Yu.B. thanks for support by the Russian program "Integration", project N. 578. This work has been supported by Academy of Finland (project "Cosmology in the Local Universe"). F.S.L. M.M. and L.P.

thank L. Amendola, H. Di Nella and A. Gabrielli for useful collaborations. F.S.L. is grateful to M. Joyce and R. Durrer for useful discussions and suggestions. 


\section{REFERENCES}

1. Baryshev, Yu.V,.Izvestiya SAO, 14, 24-43 (1981)

2. Baryshev, Yu.V, \& Teerikorpi P. The Unexpected Universe: From Harmony of Spheres to Beauty of Fractals (1998a) (in preparation)

3. Baryshev, Yu.V., \& Teerikorpi P. (1998b) (in preparation)

4. Baryshev, Yu.V., Sylos Labini F., Montuori, M., and Pietronero, L., Vistas in Astr., 38, 419-500 (1994)

5. Baryshev, Yu.V., Gravitation 2, 69-81 (1996)

6. Bondi, H., Mon.Not.R.A.S.,107,410-425 (1947)

7. Carpenter, E.F., Astrophys.J., 88, 344-355 (1938)

8. Coleman, P.H., \& Pietronero, L., Phys.Rep.231, 311-389 (1992)

9. Coles, P., Nature 391, 120-121 (1998)

10. Davis, M., in the Proc. of the Conference "Critical Dialogues in Cosmology", Ed. N. Turok, World Sci. (1997)

11. Davis, M., \& Peebles, P.J.E., Astrophys.J. 267, 465- (1983)

12. De Lapparent, V., Huchra, J. and Geller, M., Astrophys.J. 332, 44 (1988)

13. de Vaucouleurs, G., Science, 167, 1203-1213 (1970)

14. de Vega, H., Sanchez, N., and Combes, F., Nature 383, 56-58 (1996a)

15. de Vega, H., Sanchez, N., and Combes, F., Physical Review D 54, 6008 (1996b)

16. de Vega, H., Sanchez, N., and Combes, F., Astrophys. J. In the press (1998)

17. Fang, L.L,. et al. Astron.Astrophys., 243, 283-294 (1991)

18. Freedman, W., in Science with the Hubble Space Telescope - II, 3-8 Space Telescope Science Institute, eds. Benvenuti et al. , (1996)

19. Governato, F., et al. New Astronomy 2, 91-106 (1997)

20. Guzzo.. L., New Astronomy 2, 517-532 (1997) 
21. Haggerty, M. \& Wertz, J.. MNRAS 155, 495-503 (1972)

22. Harrison, E., Astrophys.J., 403, 28-31 (1993)

23. Hubble, E., Proc.Nat.A.Sci., 15, 168-173 (1929)

24. Karachentsev, I., Astron.Astrophys. 305, 33-41 (1996)

25. Karachentsev, I., Makarov, D. Astron.J. 111, 794-803 (1996)

26. Kiang, T., MNRAS, 135,1-22 (1967)

27. Layzer, D., Astron.J. 59, 268-270 (1954)

28. Lerner, E., IEEE Trans.Plasma Sci. Vol. PS-14 690-702 (1986)

29. McCrea, W.H., Astron. J. 160, 271-274 (1955)

30. Mandelbrot, B.B., Fractals:Form, Chance and Dimension, W.H.Freedman, 91977)

31. Mandelbrot, B.B., in the Proc. of the Erice Chalonge School Eds. N. Sanchez and H. de Vega, World Scientific In the press (1998)

32. Milne, E.A., Quart. J. Math. 5, 64-72 (1934)

33. Peebles, P.J.E., The Large Scale Structure of the Universe, Princeton University Press (1980)

34. Peebles, P.J.E., Publ.A.S.P., 100, 670-679 (1988)

35. Peebles, P.J.E., et al. Nature, 352, 769-776 (1991)

36. Peebles, P.J.E., Principles of Physical Cosmology, Princeton Univ.Press, (1993)

37. Pietronero, L., Physica A,144,257-284 (1987)

38. Pietronero, L., Montuori, M. and Sylos Labini, F. Proc. of the Conference "Critical Dialogues in Cosmology", Ed. N. Turok World Sci. (1997)

39. Robertson, H.P,. Publ.A.S.P., 67, 82-98 (1955)

40. Sandage, A., in The Deep Universe, eds. Binggeli, B., Buser, R., Springer, (1995)

41. Sandage, A,. et al. Astrophys.J. 172, 253-263 (1972)

42. Schulman, L., \& Seden, P. Astrophys.J. 311, 1-5 (1986)

43. Segal, I., Mathematical Cosmology and Extragalactic Astronomy Academic Press, New York (1976) 
44. Smith, R.W., J.Hist.Astron. 10, 133-165 (1979)

45. Sylos Labini, F., Montuori M. \& Pietronero L. Phys. Rep. 293, 61-226 (1998a)

46. Sylos Labini F., Montuori M. \& Pietronero L. New Astronomy, submitted (1998b)

47. Sylos Labini, F., Gabrielli, A., Pellegrini, S., and Montuori, M., in preparation (1998c)

48. Tammann, G.A., et al. in Science with the Hubble Space Telescope - II, 9-19, Space Telescope Science Institute, Eds. Benvenuti et al. (1996)

49. Teerikorpi, P., Ann.Rev.Astron.Astrophys 35, 101-136 (1997)

50. Teerikorpi, P., et al. Astron.Astrophys. In The Press (1998); astro-ph/9801197

51. Theureau, G., et al. Astron.Astrophys. 322, 730-746 (1997)

52. Weinberg, S., Gravitation and Cosmology, John Wiley \& Sons (1972)

53. Weinberg, S., The First Three Minutes, p.26, Basic Books, New York (1977)

54. Wertz, J,. Astrophys.J. 164, 227-236 (1971)

55. Zeldovich, Yu.B., Novikov, I.D., Relativistic Astrophysics Vol.2 The University of Chicago Press, p. $97(1984)$ 


\section{TABLES}

TABLE I. The volume limited samples, constructed from redshift surveys and needed in the analysis, are characterized by the following parameters: - $R_{s}(M p c)$ is the depth of the catalogue $-\Omega$ is the solid angle $-R_{1}(M p c)$ is the radius of the largest sphere that can be contained in the catalogue volume. This gives the limit of statistical validity of the sample. $-r_{0}(M p c)$ is the length at which $\xi(r) \equiv 1$. - D is the fractal dimension of the sample. The value of $r_{0}$ refers to the deepest subsample. The CfA2 and SSRS2 data are not yet available (distances are expressed in $M p c$ and $\left.H_{0}=55 \mathrm{~km} / \mathrm{sec} / \mathrm{Mpc}\right)$.

\begin{tabular}{|c|c|c|c|c|c|}
\hline Sample & $\Omega(s r)$ & $R_{s}$ & $R_{1}$ & $r_{0}$ & $D$ \\
\hline CfA1 & 1.83 & 160 & 40 & 12 & $1.9 \pm 0.2$ \\
\hline CfA2 & 1.23 & 260 & 60 & 20 & $\approx 2.0$ \\
\hline $\mathrm{PP}$ & 0.9 & 260 & 60 & 20 & $2.0 \pm 0.1$ \\
\hline SSRS1 & 1.75 & 240 & 70 & 24 & $2.0 \pm 0.1$ \\
\hline SSRS2 & 1.13 & 300 & 100 & 30 & $\approx 2.0$ \\
\hline Stromlo-APM & 1.3 & 200 & 60 & 20 & $2.2 \pm 0.1$ \\
\hline LEDA & $4 \pi$ & 600 & 300 & 90 & $2.1 \pm 0.2$ \\
\hline LCRS & 0.12 & 1000 & 36 & 12 & $1.8 \pm 0.2$ \\
\hline IRAS $1.2 J y$ & $4 \pi$ & 160 & 80 & 9 & $2.0 \pm 0.1$ \\
\hline ESP & 0.006 & 1400 & 20 & 10 & $1.9 \pm 0.2$ \\
\hline
\end{tabular}




\section{FIGURES}

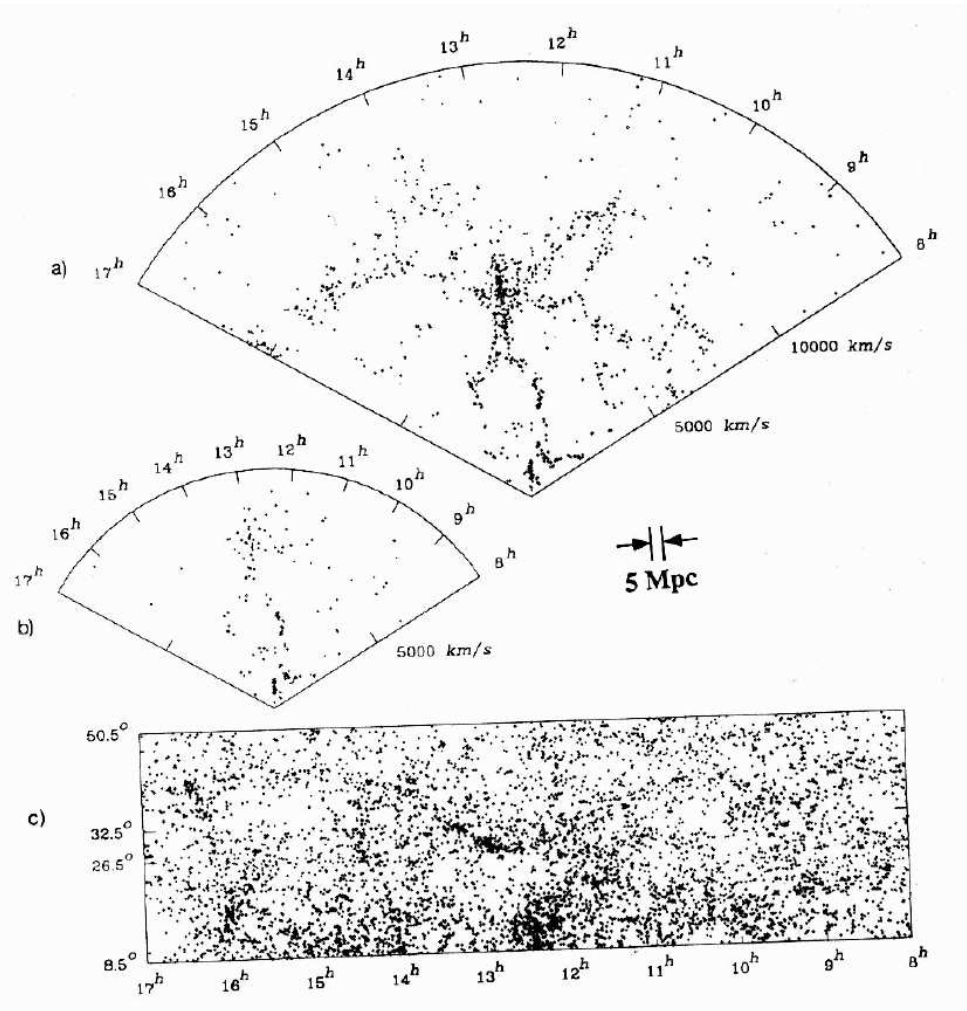

FIG. 1. A slice of the three dimensional galaxy distribution (old CfA1 catalog (a) and new CfA catalog (b)) compared with the corresponding (c) angular distribution (the portion between $26.5^{\circ}$ and $32.5^{\circ}$ ) - from the de Lapparent et al. 1988. Note that the angular distribution appears relatively homogeneous while the real distribution in space is much more irregular. In particular this picture points out the so-called Great Wall which extends over the entire sample (at least $340 \mathrm{Mpc}$ ). We also show the size of the galaxy "correlation-length" $\left(r_{0}=10 \mathrm{Mpc}\right)$ derived from the standard analysis (here distances correspond to the value of the Hubble constant $H_{0}=55 \mathrm{~km} / \mathrm{sec} / \mathrm{Mpc}$ ). The more general analysis that we discuss in the text implies instead that an eventual correlation length should be larger than any observable structure, i.e. $>340 M p c$ and that the present data show well defined fractal properties up to the sample limits (from De Lapparent et al. , 1998). 


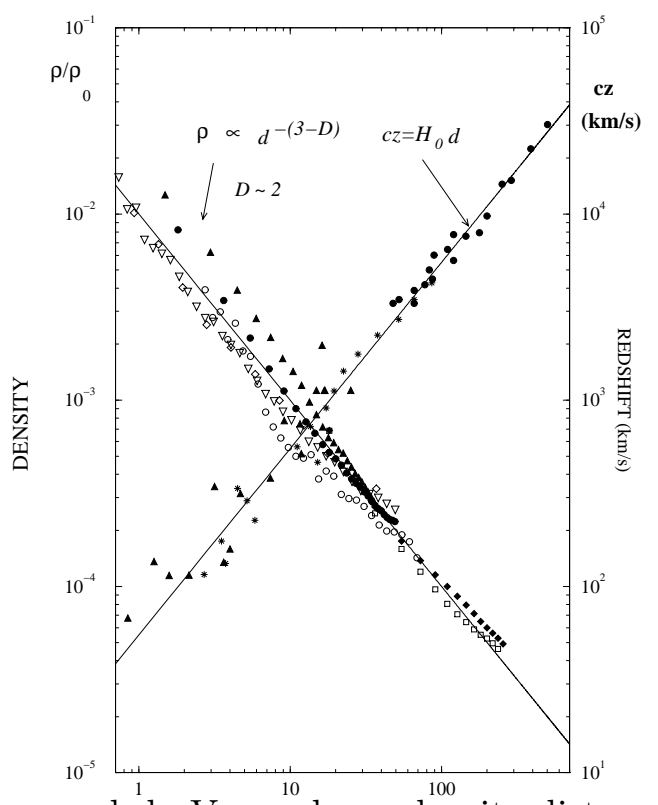

FIG. 2. Hubble redshift-distance and de Vaucouderars density-distance laws in the distance scales from 1 to $500 M p c\left(H_{0}=55 \mathrm{~km} / \mathrm{s} / \mathrm{Mpc}\right)$. The Hubble law (increasing from left to right) is constructed from: galaxies with Cepheid-distances for $c z>0$ (triangles), galaxies with Tully-Fisher (B-magnitude) distances (stars), galaxies with SNIa-distances for $c z>3000 \mathrm{~km} / \mathrm{s}$ (filled circles). TF-distance points are generally averages of a few tens of galaxies from the "unbiased plateau" of the method of normalized distances. Redshift $c z$ is reduced to the Local Group center and contains the small correction due to the Virgo infall velocity field. The solid line corresponds to the Hubble law with $H_{o}=55 \mathrm{~km} / \mathrm{s} / \mathrm{Mpc}$. The de Vaucouleurs law (decreasing from left to right) in the normalized form is constructed from the computation of the conditional average density in the following redshift surveys: CfA1 (crosses), Perseus-Pisces, LCRS (filled diamonds), ESP (triangles left) and LEDA (Sylos Labini et al. , 1998). The normalization between the different densities takes into account the different magnitude limited of the various redshift surveys. The dotted line corresponds to the de Vaucouleurs law with correlation exponent $\gamma=1$, i.e. $D=2$. 


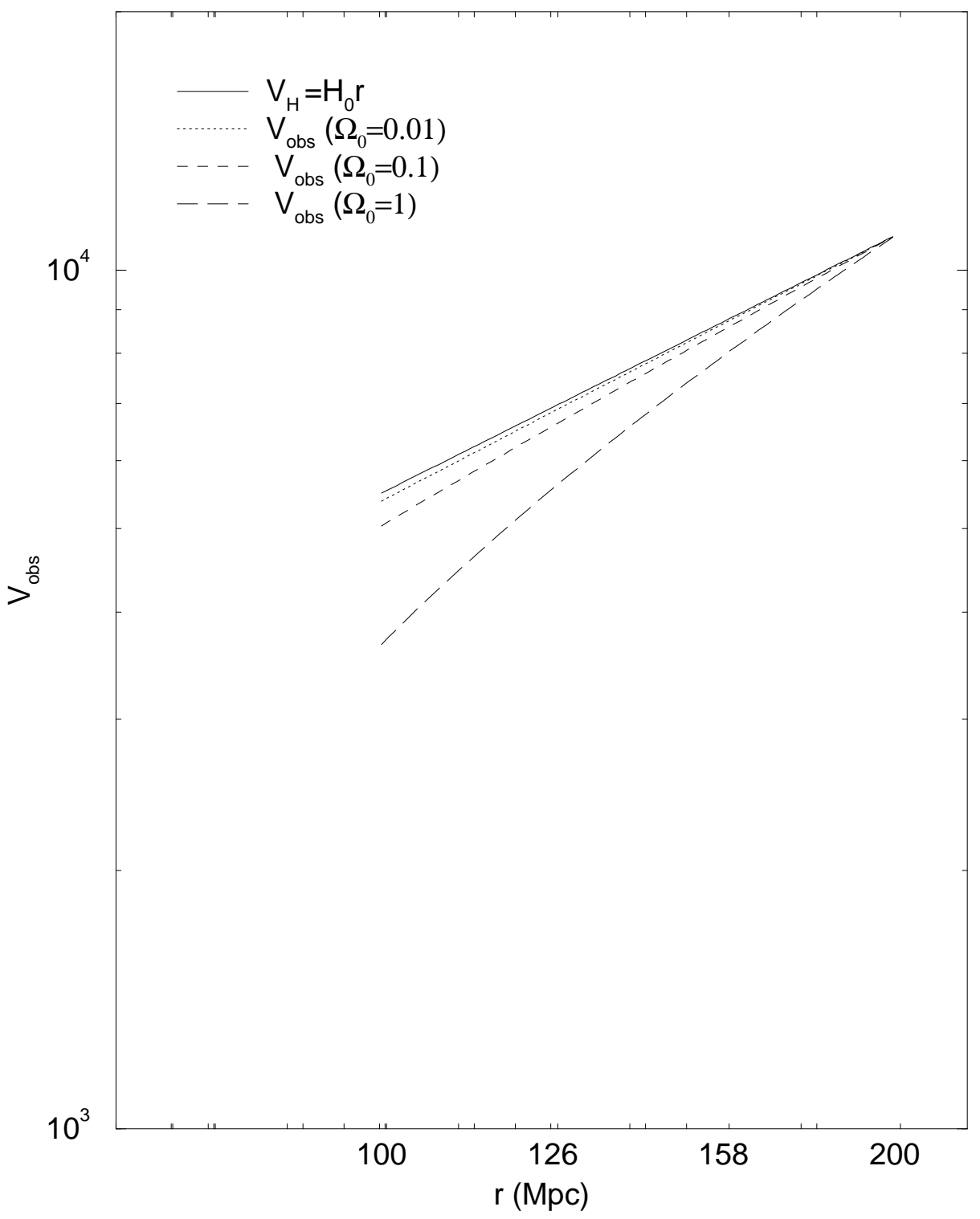

FIG. 3. We show three theoretical predictions for the velocity deflection in the case where the observed fractal structure contains all the matter, i.e. when $\Omega_{l u m}=\Omega_{0}$. We have fixed $\lambda_{0}=200 M p c$ and fractal dimension $D=2$. The predictions correspond to three values of the cosmological density parameter $\Omega_{0}=1,0.1,0.01$. 


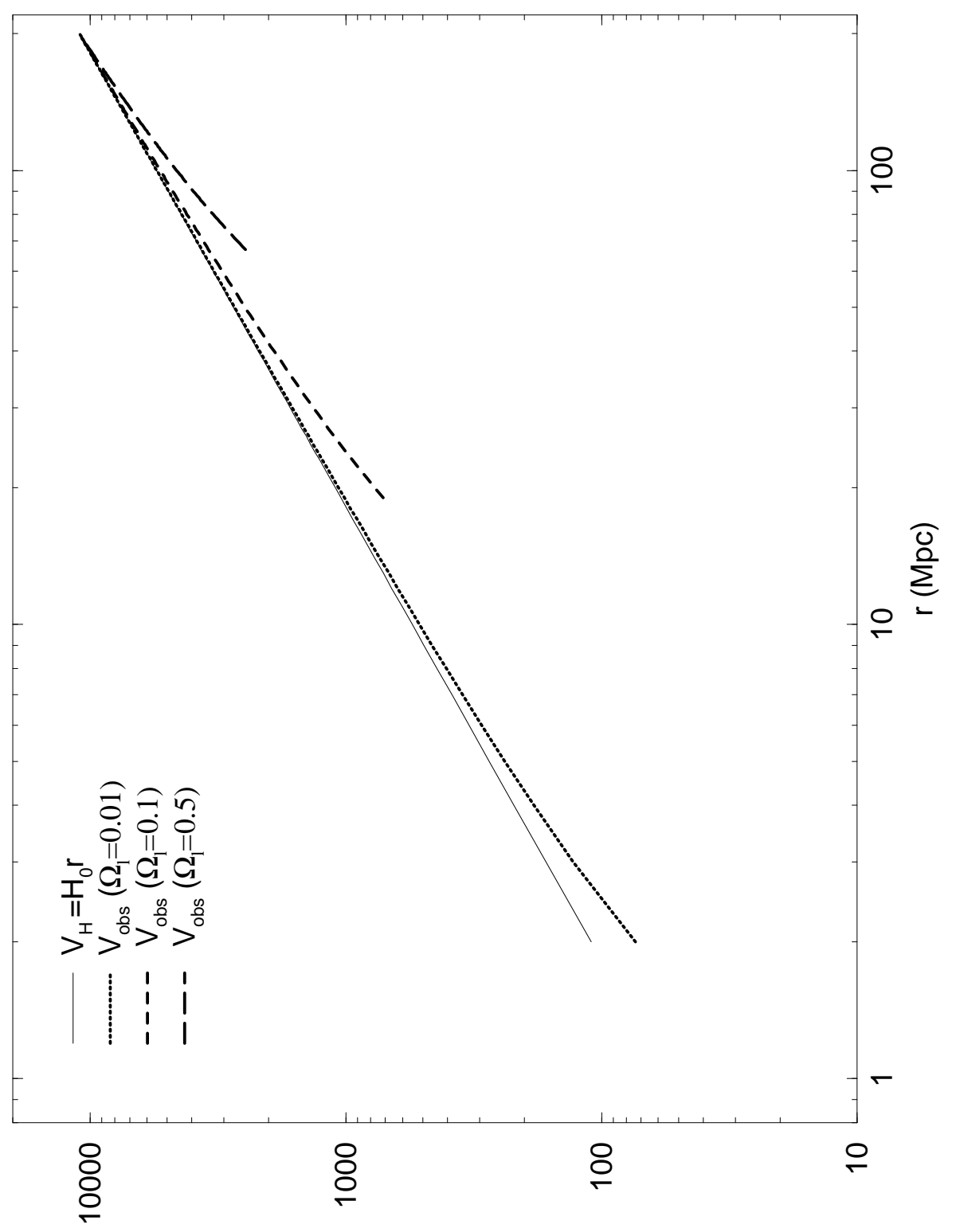

FIG. 4. In this case the total density is $\Omega_{0}=1$. sad $X$ e show the behaviour of $V_{o b s}$ derived by the linear perturbation approximation in the case $\delta \rho / \rho_{0} \ll 1$, for various values of $\Omega_{\text {lum }}$. The fractal dimension of luminous matter is $D=$ up to $\lambda_{0}=200 M p c$. 